\title{
The LIM-only transcription factor LMO2 determines tumorigenic and angiogenic traits in glioma stem cells
}

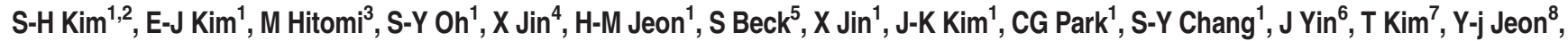 \\ J Song ${ }^{2}$, YC Lim ${ }^{9}$, JD Lathia, ${ }^{3,10,11}$, I Nakano ${ }^{\star, 2,12}$ and H Kim ${ }^{\star, 1}$
}

Glioblastomas (GBMs) maintain their cellular heterogeneity with glioma stem cells (GSCs) producing a variety of tumor cell types. Here we interrogated the oncogenic roles of Lim domain only 2 (LMO2) in GBM and GSCs in mice and human. High expression of LMO2 was found in human patient-derived GSCs compared with the differentiated progeny cells. LMO2 is required for GSC proliferation both in vitro and in vivo, as shRNA-mediated LMO2 silencing attenuated tumor growth derived from human GSCs. Further, LMO2 is sufficient to induce stem cell characteristics (stemness) in mouse premalignant astrocytes, as forced LMO2 expression facilitated in vitro and in vivo growth of astrocytes derived from Ink4a/Arf null mice and acquisition of GSC phenotypes. A subset of mouse and human GSCs converted into vascular endothelial-like tumor cells both in vitro and in vivo, which phenotype was attenuated by LMO2 silencing and promoted by LMO2 overexpression. Mechanistically, the action of LMO2 for induction of glioma stemness is mediated by transcriptional regulation of Jagged1 resulting in activation of the Notch pathway, whereas LMO2 directly occupies the promoter regions of the VE-cadherin gene for a gain of endothelial cellular phenotype. Subsequently, selective ablation of human GSC-derived VE-cadherin-expressing cells attenuated vascular formation in mouse intracranial tumors, thereby significantly prolonging mouse survival. Clinically, LMO2 expression was elevated in GBM tissues and inversely correlated with prognosis of GBM patients. Taken together, our findings describe novel dual roles of LMO2 to induce tumorigenesis and angiogenesis, and provide potential therapeutic targets in GBMs.

Cell Death and Differentiation (2015) 22, 1517-1525; doi:10.1038/cdd.2015.7; published online 27 February 2015

Glioblastoma (GBM) is the most frequent and lethal primary brain tumor with inevitable recurrence in the vast majority of cases after conventional therapy. ${ }^{1}$ Therefore, there is an urgent need to develop novel therapeutic options that effectively target therapy-resistant GBM cells. Cancer stem cells in GBM (glioma stem cells: GSCs) are a subpopulation of tumor cells that retains undifferentiated stem cell characteristics (stemness) and high tumorigenic potential. ${ }^{2}$ Evidence is accumulating that GSCs drive GBM initiation and propagation and contribute to the development of resistance to current treatment options. ${ }^{3-6}$ Therefore, this provides a novel therapeutic rationale for targeting GSC in GBM. However, the clinical significance of GSCs is still controversial and the regulatory molecular mechanisms for GSCs remain elusive.

The LMO2 gene contains two zinc-binding LIM-domains that are essential for LMO2 as a bridging molecule in multiprotein complexes. ${ }^{7}$ Through binding of the LIM domain to various proteins including TAL/SCL, GATA-1, E47, and
LDB1, they are able to regulate gene expression at the transcriptional level by recognizing a unique bipartite DNA sequence comprising an $E$ box separated by about one helix turn from a GATA site. ${ }^{8}$

Transcriptional dysregulation of $L M O 2$ is frequently observed in human acute T-cell lymphoblastic leukemia patients. ${ }^{9} \mathrm{Lmo} 2$ transgenic activation in the thymus results in T-cell lymphoma/leukemia. Lmo2 overexpression in T-cell progenitors caused differentiation block, exit from quiescence, and increased self-renewal, all of which are the hallmarks of hematopoietic stem cells (HSCs). ${ }^{10}$ Indeed, Lmo2-expressing T-cell progenitors display an HSC-like transcriptional signature indicating de-differentiation. This reprogramming event caused by $\mathrm{Lmo} 2$ may indicate that LMO2 is a driver of cancer initiation in T-cell progenitors.

Despite these intensive studies of LMO2 in leukemia genesis in the past decade, pathophysiology of LMO2 in solid cancers remains largely undetermined. In this study, we

\footnotetext{
${ }^{1}$ School of Life Sciences and Biotechnology and Institute of Life Science and Natural Resources, Korea University, Seoul 136-713, Republic of Korea; ${ }^{2}$ Department of Neurological Surgery, The Ohio State University, Columbus, OH 43210, USA; ${ }^{3}$ Department of Cellular and Molecular Medicine, Lerner Research Institute, Cleveland Clinic, Cleveland, OH 44195, USA; ${ }^{4}$ Department of Stem Cell Biology and Regenerative Medicine, Lerner Research Institute, Cleveland Clinic, Cleveland, OH 44195, USA; ${ }^{5}$ Department of Molecular Biosciences, University of Texas at Austin, Austin, TX 78712, USA; ${ }^{6}$ Specific Organs Cancer Branch, Research Institute and Hospital, National Cancer Center, Goyang 410-769, Republic of Korea; ${ }^{7}$ Department of Molecular and Cellular Oncology, The University of Texas MD Anderson Cancer Center, Houston, TX 77030, USA; ${ }^{8}$ Department of Molecular Virology, Immunology and Medical Genetics, The Ohio State University, Columbus, OH 43210, USA; ${ }^{9}$ Department of Otorhinolaryngology-Head and Neck Surgery, Research Institute of Medical Science, Konkuk University School of Medicine, Seoul 143-752, Republic of Korea; ${ }^{10}$ Department of Molecular Medicine, Cleveland Clinic Lerner College of Medicine, Cleveland, OH 44195, USA; ${ }^{11}$ Case Comprehensive Cancer Center, Cleveland, OH 44195, USA and ${ }^{12}$ James Comprehensive Cancer Center, The Ohio State University, Columbus, OH 43210, USA

*Corresponding authors: I Nakano, Department of Neurological Surgery, The Ohio State University, 410 w 12th avenue, Columbus 43210, OH, USA. Tel: +1 614 292 0358; Fax: +1 614688 4882; E-mail: Ichiro.Nakano@osumc.edu

or H Kim, School of Life Sciences and Biotechnology and Institute of Life Science and Natural Resources, Korea University, Seoul 136-713, Republic of Korea. Tel: +82 23290 3059; Fax: +82 23290 3040; E-mail: hg-kim@korea.ac.kr

Abbreviations: GBM, glioblastoma; GFAP, glial fibrillary acidic protein; GSCs, glioma stem cells; HSCs, hematopoietic stem cells; LMO2, Lim domain only 2; NBE, neurobasal medium

Received 26.6.14; revised 30.12.14; accepted 14.1.15; Edited by R De Maria; published online 27.2.15
} 
sought to elucidate the physiological roles and mechanism of action of LMO2 in GBM and GSCs in mice and human.

\section{Results}

LMO2 is required for GSC growth both in vitro and in vivo. First, we investigated the expression of LMO2 in GSC and non-GSC derived from acutely dissociated primary xenografts propagated in vivo sorted for CD133-positive and -negative population. Real-time PCR and western blot analysis demonstrated that LMO2 expression was reduced, whereas an astrocyte differentiation marker, glial fibrillary acidic protein was increased in the non-GSCs in Figure 1a. Human GBM-derived GSCs showed significant decline of LMO2 expression upon induction of differentiation with serum-containing media (Supplementary Figure 1a). Transcriptome microarray data with 11 GCS samples and 5 normal astrocyte samples from Mao data set demonstrated that human GSCs have relatively higher LMO2 mRNA expression compared with differentiated normal astrocytes (Figure 1b). Elevated LMO2 expression in GSCs was also observed in two other data set (Schulte data set and Lee data set; Figure 1c and Supplementary Figure 1b). Among the two GSC subtypes, LMO2 expression was specifically higher in proneural subtype than mesenchymal one in both Mao data set and Bhat data set (Figure 1d). Altogether, LMO2 expression is enriched in GSCs with proneural identity. We then performed LMO2 knockdown by five different shLMO2 lentivirus clones in GSCs and selected the shLMO2 \#5 with $90 \%$ reduction of LMO2 for the further study (Supplementary Figure $2 \mathrm{a}$ and $\mathrm{b}$ ). In vivo limiting dilution assay shows that LMO2 depletion attenuated sphere-forming activity in GSC1T (Figure 1e and Supplementary Figure 2c). Conversely, LMO2 overexpression exhibited increased sphere-forming activity in the non-GSC, U87MG (Supplementary Figure 2d and e). These in vitro data appeared relevant in vivo, as the orthotopic mouse xenograft assays revealed that tumor growth of human GSCs is significantly diminished by LMO2 knockdown (Figure 1f).

LMO2 is sufficient to induce glioma stemness from premalignant astrocytes both in vitro and in vivo. Next we investigated the phenotypic consequences of Lmo2 overexpression in primary premalignant astrocytes derived from Ink4a/Arf null mice (hereby termed as Ink4a/Arf ${ }^{-1-}$ astrocytes), which lack p16 ${ }^{\text {lnk4a }}$ and $\mathrm{p} 19^{\text {Arf }}$ genes-the most frequently altered tumor suppressor genes in human GBM. ${ }^{13}$ Overexpression of $L m o 2$ increased in vitro growth of Ink4a/ $\mathrm{Arf}^{-1-}$ astrocytes (Figure 2a). This increase of cell growth by Lmo2 overexpression was associated with elevated expression of CyclinD1 protein (Figure 2b). Consistent with these in vitro data, Lmo2 overexpression significantly accelerated in vivo growth of subcutaneous and intracranial mouse tumors derived from Ink4a/Arf ${ }^{-1-}$ astrocytes (Figure 2c). Notably, Lmo2- Ink4a/Arf ${ }^{-1}$ astrocytes-derived tumors have a

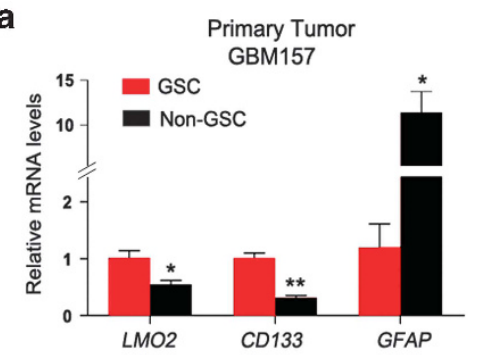

d
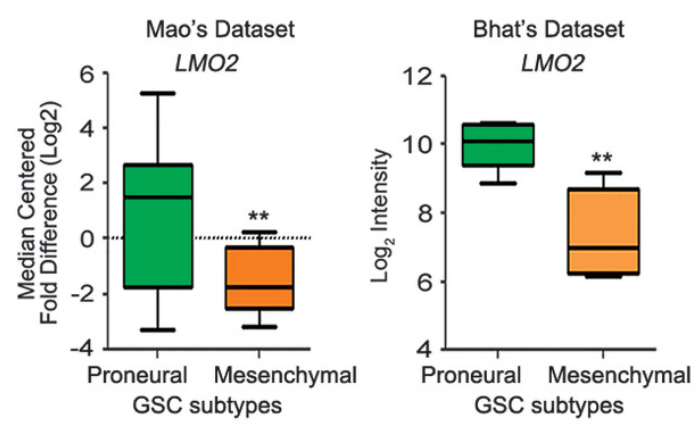

b

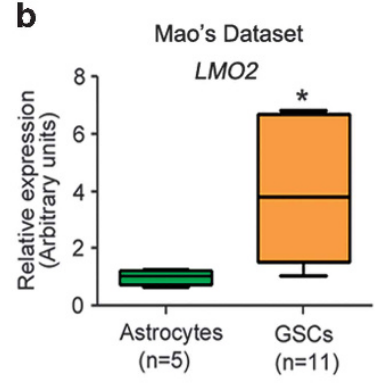

e

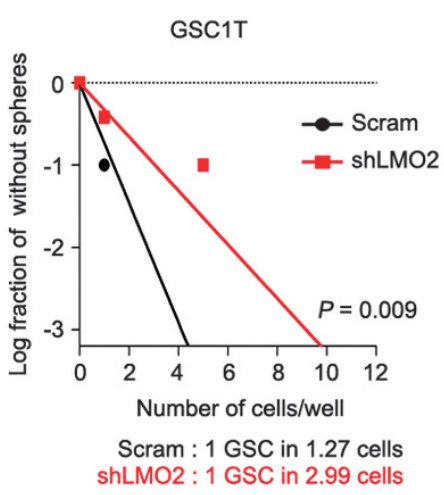

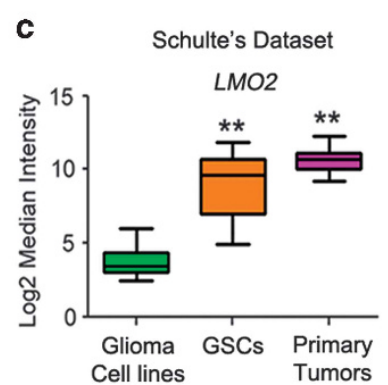

f

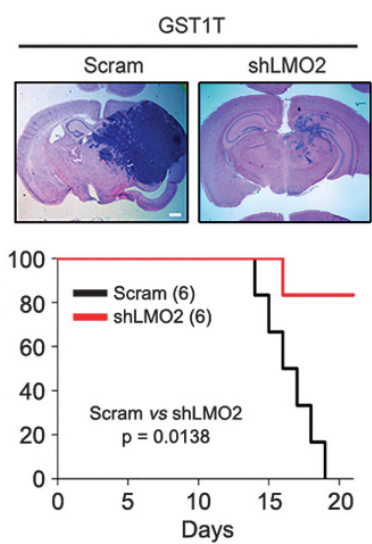

Figure 1 LMO2 is necessary for human glioma stem cell growth in vivo (a) mRNA and protein expression of LMO2, CD133, and glial fibrillary acidic protein (GFAP) in GSC and non-GSC acutely isolated from two different GBM-derived xenograft brain tumors based on the CD133 expression. (b and $\mathbf{c}$ ) Relative LMO2 mRNA expression in indicated samples in Mao's data set (b) and Schulte's data set (c). (d) Relative LMO2 expression in proneural and mesenchymal GSC samples in indicated data set. (e) In vitro limiting dilution assays (LDAs) to determine the effect of shLMO2 on self-renewal activity in GSC1T cells. Wells without sphere formation were counted. $P=0.009$ with extreme LDA (ELDA) analysis; http:// bioinf.wehi.edu.acu/software/elda/. (f) Representative H\&E staining of whole-mouse brain xenografts derived from Scram- and shLMO2-GSCs (GST1T) and the Kaplan-Meier survival analysis. Scale bar represents $500 \mu \mathrm{m}$. Asterisk ( $\left.{ }^{*}\right)$ indicates statistical significance by Student's $t$-test. ${ }^{*} P<0.05 ;{ }^{* \star} P<0.01$ 
a

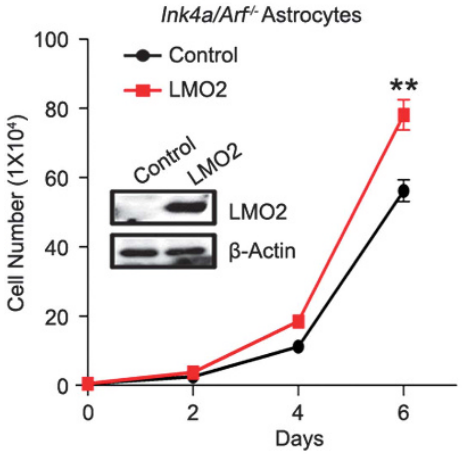

b

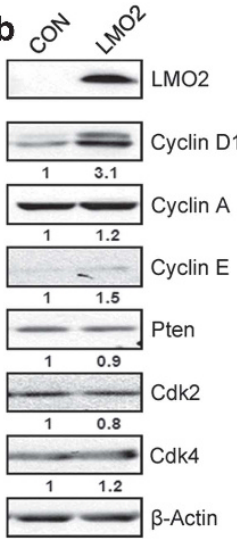

c

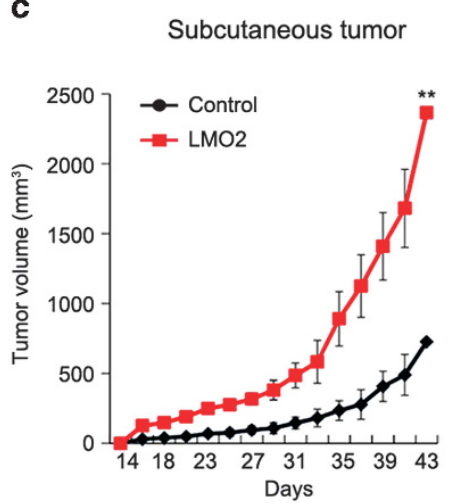

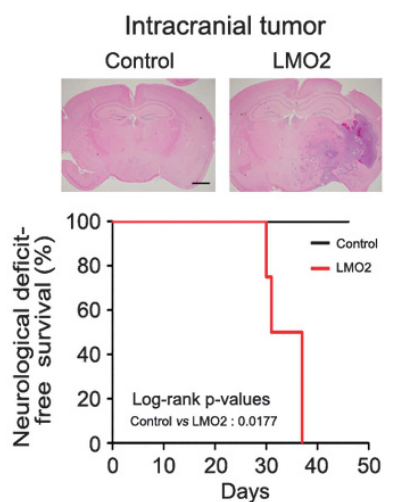

d
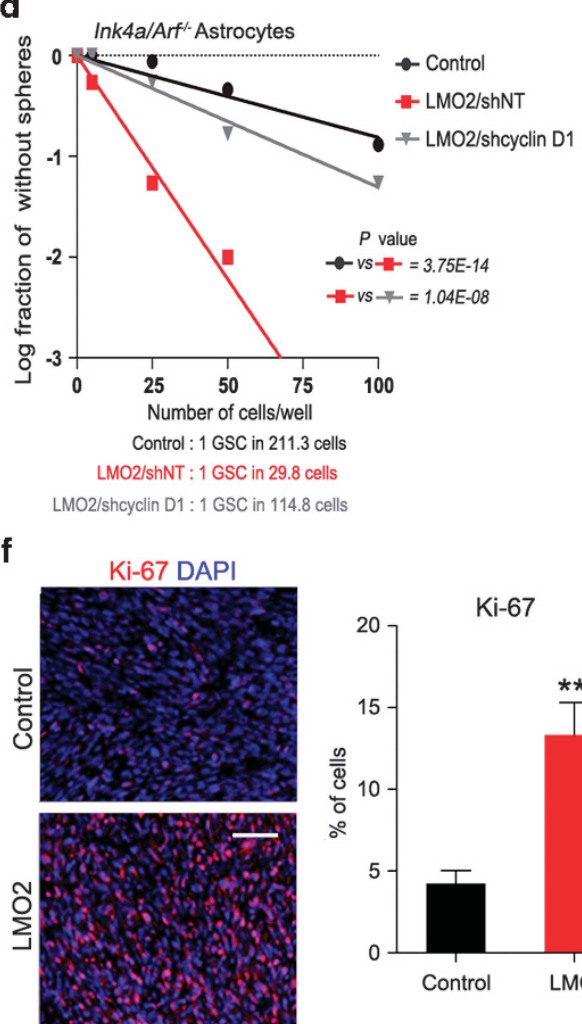
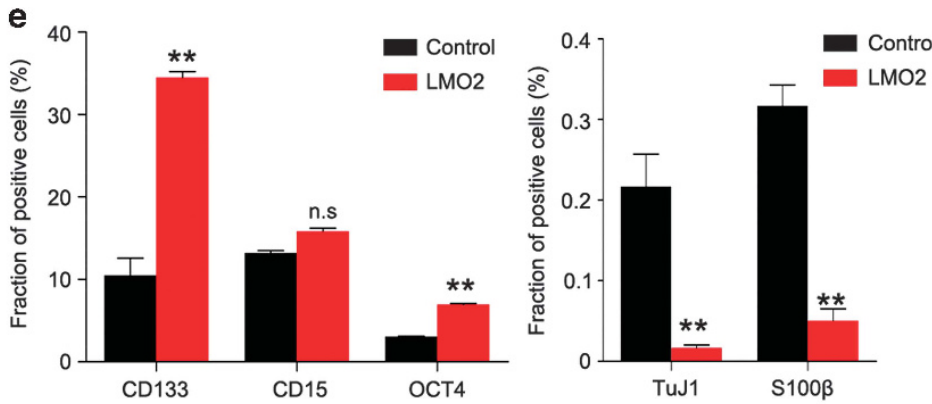

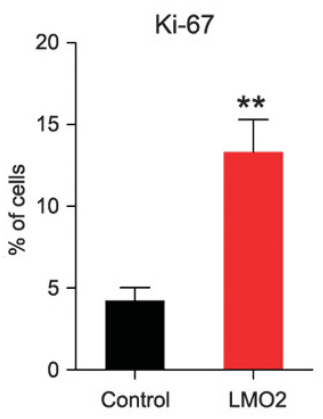

9

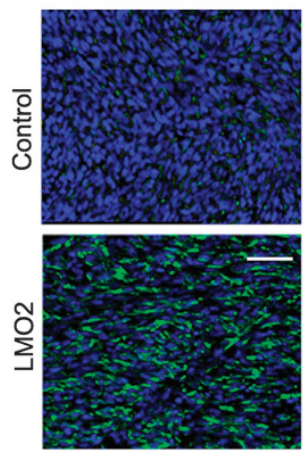

CD133 DAPI

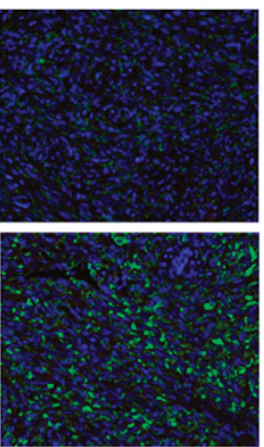

S100ß DAP

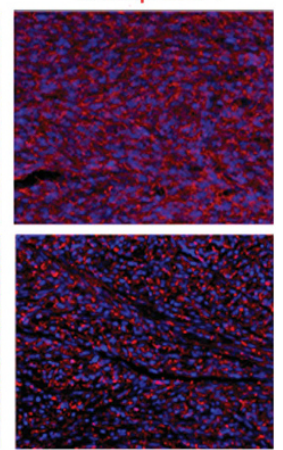

Figure 2 LMO is sufficient to induce stem cell phenotype in mouse premalignant astrocytes in vivo (a) Cell proliferation of Control-, and Lmo2-overexpressing Ink4a/Arf ${ }^{\prime-}$ astrocytes in vitro. Western blot showing the expression level of LMO2 in each sample. $\beta$-actin as internal control. (b) Protein expression levels of cell cycle-associated proteins in indicated samples. (c) An in vivo mouse subcutaneous ( $n=6$ per group, left panel) and intracranial ( $n=4$ per group, right upper panel) tumorigenic potential of Control- and Lmo2-Ink4a/Arf ${ }^{-1-}$ astrocytes. Kaplan-Meier analysis of neurological deficit-free survival for the Lmo2 overexpression compared with the control (right lower panel). Scale bar represents $500 \mu \mathrm{m}$. (d) In vitro limiting dilution assay of Control-, Lmo2-, and Lmo2/shCyclin D1-Ink4a/Arf ${ }^{-1-}$ astrocytes. (e) Proportion of CD133(+), CD15(+), OCT4(+), TuJ-1(+), and $\mathrm{S} 100 \beta(+)$ cells of Control- and $\mathrm{Lmo2}_{\mathrm{I}} \mathrm{nk} 4 \mathrm{a} / \mathrm{Arf}^{-1-}$ astrocytes. (f) Representative immunofluorescence images and quantification of Ki-67 in subcutaneous tumors derived from Control- and Lmo2-Ink4aArf-/ - astrocytes. (g) Representative immunofluorescence images of Nestin, CD133, and S100 $\beta$ expression in subcutaneous tumors derived from Control- and Lmo2-Ink4aArf- / - astrocytes. DAPI for nuclear staining. Scale bars represent $100 \mu \mathrm{m}$. Data are expressed as mean \pm S.D. ${ }^{* *} P<0.01$

been shown to have histological features of high-grade glioma, such as intratumoral hemorrhage, angiogenesis, and necrosis (Supplementary Figure 3).

Because Lmo2 promotes self-renewal of preleukemic thymocytes, ${ }^{10}$ we investigated if Lmo2 overexpression leads to stem cell phenotypes in brain cancers. When we performed in vitro limiting dilution assay, Lmo2-overexpressing Ink4a/ $\mathrm{Arf}^{-1}$ astrocytes promotes sphere-forming cells that are markedly decreased by Cyclin $D 1$ knockdown, suggesting Cyclin D1 is involved in Lmo2 mediated self-renewal activity (Figure 2d). With regard to the marker expression, Lmo2overexpressing cells displayed a marked increase of stem cell marker, CD133; ${ }^{14} 8 \%$ versus $35 \%$, and OCT $4 ; 3 \%$ versus $7 \%$ of CD133(+) cells in control versus Lmo2-ovexpressing cells as determined by FACS analysis (Figure 2e). In turn, expression of the differentiation markers, TuJ1 and $\mathrm{S} 100 \beta$, 
was strongly decreased in Lmo2-overexpressing cells. Consistent with these in vitro data, immunohistochemistry demonstrated increases of Ki-67, Nestin(+), and CD133(+) cells in Lmo2-overexpressing subcutaneous mouse tumors (Figure $2 \mathrm{f}$ and $\mathrm{g}$ ). On the other hand, these tumors diminished the proportion of $\mathrm{S} 100 \beta(+)$ cells (Figure $2 \mathrm{~g}$ ). Taken together, Lmo2 promotes tumor propagation by inducing undifferentiated stem cell phenotype of Ink4a/Arf ${ }^{-1-}$ astrocytes.

\section{LMO2 mediates GSC phenotype through transcriptional} regulation of the Jagged1-Notch signaling axis. Previously we identified that the key signaling pathways activated in proneural GSCs include Notch-, Shh-, and Wntmediated pathways. ${ }^{12}$ Given that LMO2 is highly expressed in proneural but not mesenchymal GSCs (Figure 1d), we analyzed the effect of $L M O 2$ on these signaling pathways using luciferase reporter vectors containing the specific binding sequences of either CSL (Notch signaling), Gli-1 (Shh signaling), or TCF/LEF (TOP; Wnt/ $\beta$-catenin signaling). Lmo2 overexpression resulted in a 1.8-fold increase of the CSL/Notch-responsive promoter activity (Figure 3a). Quantitative RT-PCR revealed that one of the Notch ligands, Jagged1, is upregulated in Lmo2-Ink4a/Arf ${ }^{-/-}$astrocytes (Figure 3b). Jagged1 promoter region has one E-box (CANNTG) DNA motif that is known as a binding site for the LMO2 transcriptional complexes. ${ }^{15}$ Indeed, Lmo2 increased Jagged1 promoter activity in a dose-dependent manner (Figure 3c), and Jagged1 protein and cleaved Notch1 (Notch intracellular domain-NICD; Figure $3 d$ and Supplementary Figure 4a). Lmo2 also increased Notch downstream target genes Hes1 and Hey1, and these increases were completely attenuated by shRNA-mediated depletion of Jagged1 (Figure $3 e$ and Supplementary Figure $4 \mathrm{~b})$. In turn, depletion of $L M O 2$ by shRNA greatly reduced Jagged1 expression in human GSCs (Figure 3f). LMO2 expression in GSCs exhibited statistically significant positive correlation with those of Jagged1, Hes1, Hey1, and Hey2 (Supplementary Figure 5). In addition, a gene set enrichment analysis (GSEA) of transcriptome in GBM patients exhibited a significant correlation between $\mathrm{LMO} 2$ and $\mathrm{NOTCH}$ target gene signature (Figure $3 \mathrm{~g}$ ). The sphere-forming ability of Lmo2-Ink4a/Arf ${ }^{-1-}$ astrocytes was completely attenuated by Jagged1 depletion (Figure 3h and Supplementary Figure 6). Collectively, these data indicate that LMO2 causally contributes to the reprogramming of differentiated astrocytes into GSC-like tumorigenic cells in a Jagged1/Notch pathwaydependent manner.

LMO2 induces endothelial-like cells from GSCs. Next, we investigated the cellular identities of xenografted human GSCs in mouse brains by using a GFP-labeled GSC sample $\left(\mathrm{GFP}^{+} \mathrm{GSC} 1 \mathrm{~T}\right)$. With a close investigation at the perivascular area in tumors, we identified $\operatorname{VWF}(+)$ cells contained either $\mathrm{GFP}(+)$ or $(-)$ cells (Figure $4 \mathrm{a}$ ), raising a possibility that tumor vasculature is composed of both host endothelial cells and GSC-derived cells that utilize vascular mimicry. ${ }^{16}$ Given that LMO2 is widely expressed in the vasculature of a variety of neoplasm, ${ }^{17}$ we sought to determine whether the LMO2driven tumorigenicity is associated with facilitated angiogenesis in an autocrine or paracrine manner. To assess the paracrine effect, we treated human umbilical vein endothelial cells (HUVECs) with the conditioned medium derived from Lmo2-overexpressing cells under the endothelial cell growth-promoting culture condition. This treatment did not exhibit any significant changes in in vitro tube formation, suggesting that Lmo2 may not be involved in angiogenic induction in a paracrine manner (Figure $4 \mathrm{~b}$ and Supplementary Figure 7a). Next, we directly induced tube formation of Control- and Lmo2-Ink4a/Arf ${ }^{-/-}$astrocytes using the same culture condition. Lmo2 overexpression markedly increased the tube-like structure in an eightfold in Ink4a/Arf ${ }^{-1}$ - cells compared with the control (Figure $4 \mathrm{~b}$ and Supplementary Figure 7b). Furthermore, this phenotypic change by Lmo2 transduction was associated with enhanced immunoreactivity to two endothelial-specific markers, VEGFR2 and VE-cadherin (Figure 4c). Quantitative analysis by FACS showed the increases of VEGFR2, VE-cadherin, and CD34 were greater than sixfold (Figure 4d). Consistent with these mouse data, LMO2 knockdown in human primary GSCs (GSC1T) significantly reduced the number of tubes along with the decreased expression of VE-cadherin and VEGFR2 in vitro (Figure 4e,Supplementary Figure 7c and d). Furthermore, LMO2 knockdown in GFP-expressing human GSC1T brain xenografts resulted in a fivefold decrease in vWF-expressing GFP(+) cells compared with the control tumors (Figure 4f). In turn, Lmo2 overexpression displayed a substantial increase of $\operatorname{VWF}(+)$ cells in Lmo2-Ink4a/Arf ${ }^{-1-}$ astrocytes-derived mouse tumors (Figure $4 \mathrm{~g}$ ).

We next performed chromatin immunoprecipitation assay to determine whether LMO2 directly occupies the promoter regions of the VE-cadherin gene. Both of the two VE-cadherin promoter regions were significantly amplified by quantitative PCR from chromatin immunoprecipitated Ink4a/Arf ${ }^{-1-}$ astrocyte samples using LMO2 antibody in the proangiogenic condition (EGM2 media; Figure 4h). Collectively, these data indicate that $\mathrm{LMO} 2$ binds and activates the promoter regions of the VE-cadherin gene thereby promoting endothelial-like cells from mouse premalignant astrocytes and human GSCs both in vitro and in vivo.

VE-cadherin(+) cells derived from GSCs promotes tumor aggressiveness. We then sought to determine whether the human GSC-derived VE-cadherin(+) cells contribute to tumor growth in vivo (Figure 5). To this end, we transplanted GSCs after infection with a lentiviral vector containing the herpes simplex virus thymidine kinase gene $(t k)$ under the control of transcriptional regulatory elements of the VE-cadherin gene (VE-cadherin-tk) so that the GSC-derived VE-cadherin(+) cells would be selectively eradicated by ganciclovir. The ganciclovir treatment markedly diminished the $\operatorname{vWF}(+)$ cell population in VE-cadherin-tk expressing mouse tumors but not in the negative control tumors (Figure 5a). As a positive control (PGK-tk), we validated the cytotoxic effect of ganciclovir in PGK-tk expressing tumors. Consequently, elimination of GSC-derived VE-cadherin-expressing cells resulted in a statistically significant survival benefit for mice bearing GBM-like tumors derived from GSCs (Figure 5b).

Clinical relevance of LMO2 as a therapeutic target in GBM. Last, we examined the clinical relevance of these 


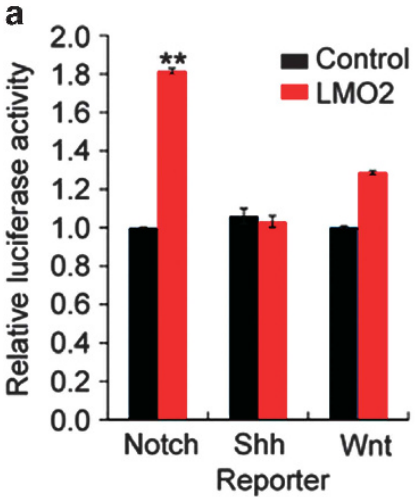

d

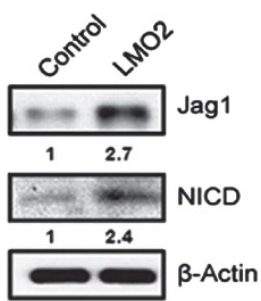

b

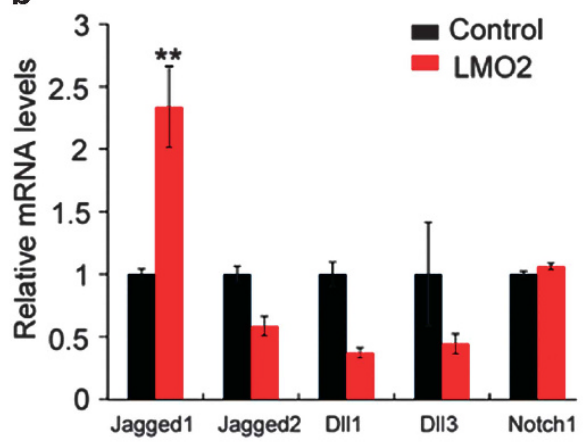

e

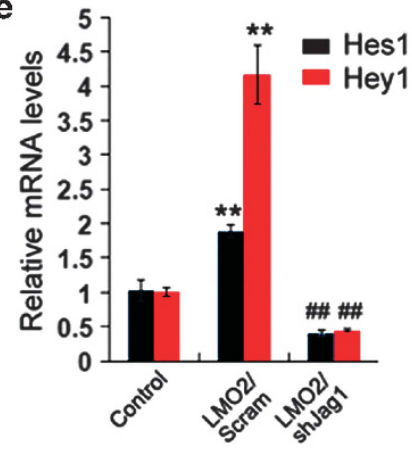

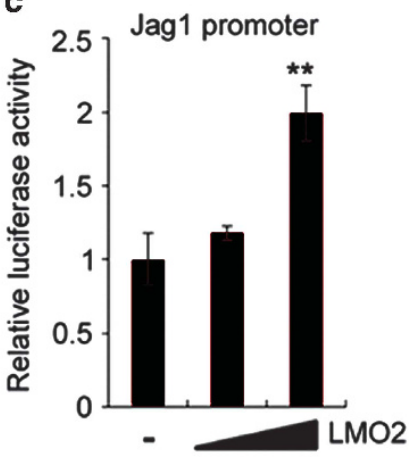

f

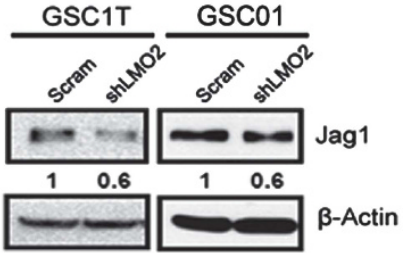

g

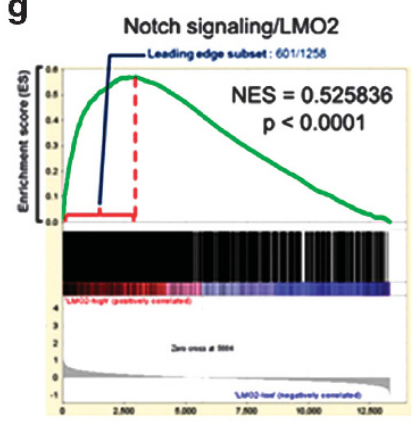

h

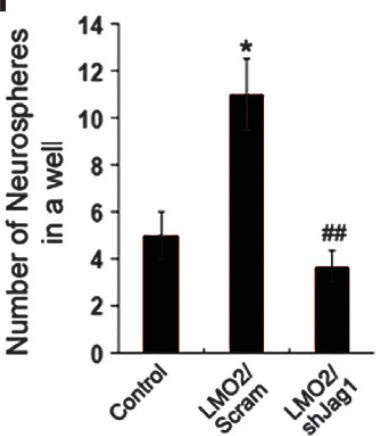

Figure 3 LMO2 maintains stem cell phenotypes by transcriptional activation of Jagged1-Notch signaling pathway (a) Relative activity of Notch (CLS), Shh (GLI-1), and Wnt (TCF-LEF) responses element luciferase reporters. (b) Relative mRNA levels of Jagged1, Jagged2, DII1, DII3, and Notch1. (c) The effect of LMO2 overexpression on the Jagged1 promoter-driven luciferase activity. Rectangle indicates increasing doses of the LMO2 expressing vector. (d) Western blot showing expression levels of Jagged1 and active Notch1 (NICD) proteins in indicated samples. (e) Hes1 and Hey1 mRNA levels of Control-, Lmo2-, and Lmo2-shJagged1-Ink4a/Arf ${ }^{-1-}$ astrocytes. (f) Jagged1 protein levels in 2 GSC samples with or without LMO2 silencing (shLMO2). (g) GSEA analysis showing the high enrichment of Notch target gene signature in LMO2-overexpressng tumors. (h) The numbers of neurosphere forming cells in indicated samples. Data are mean \pm S.D. ${ }^{*} P<0.05,{ }^{\star \star} P<0.01,{ }^{\# \#} P<0.01$

experimental data by using the glioma tumor samples. In order to do that, we interrogated tissue microarray of 93 clinical glioma samples containing WHO grade II-IV tumors, as well as adjacent normal brain tissues to determine its expression and a potential contribution of LMO2 to the patient survival. Immunohistochemistry displayed significantly higher LMO2 protein expression in high-grade glioma tissues compared with low-grade glioma tissues (Figure 6a and Supplementary Figure 8a). Furthermore, the expression of LMO2 is correlated with poor GBM patient survival (Supplementary Figure 8b). Among the 4 LMO genes, LMO2 was significantly elevated in astrocytoma, oligodendrocytoma, and GBM compared with nontumor tissues as analyzing by the Repository of Molecular Brain Neoplasia Data (REMBRANT) and Grzmil's data set (Figure $6 \mathrm{~b}$ and Supplementary Figure 8c). Significant elevation of LMO2 expression in GBM tumors compared with lower grade glioma tissues was also confirmed with two different data set (Phillips and Freije; Figure $6 \mathrm{c}$ ). When GBM patients were separated based on the prognosis (cut off: 3-year survival), those with shorter survival had significantly elevated LMO2 expression (Figure 6d). This trend was also confirmed by the Kaplan-Meier survival analysis (Figure 6e and Supplementary Figure 7d; REMBRANT survival data). Interestingly, higher LMO2 levels were also strongly correlated with patient clinical characteristics such as age ( $>40$ years), histopathological grade (WHO grade 

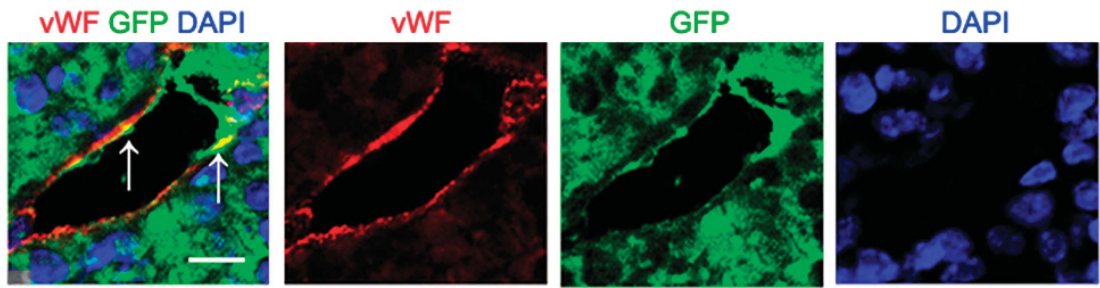

b

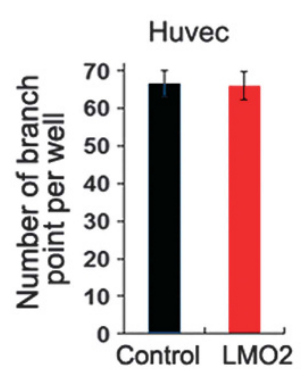

Ink4a/Arf/- Astrocytes
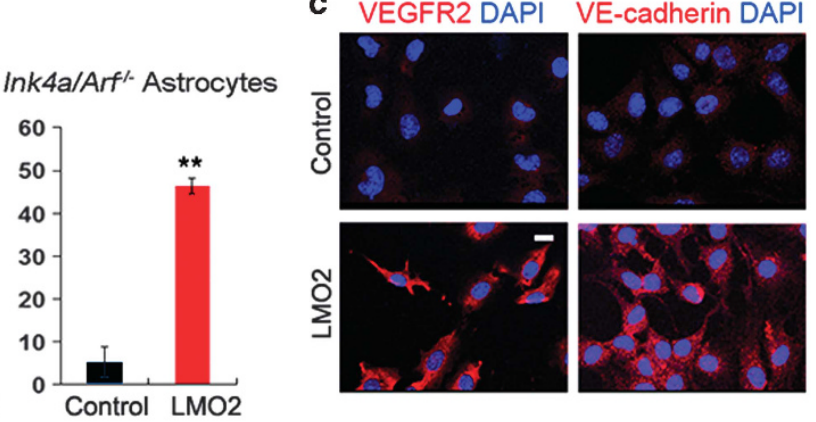

d

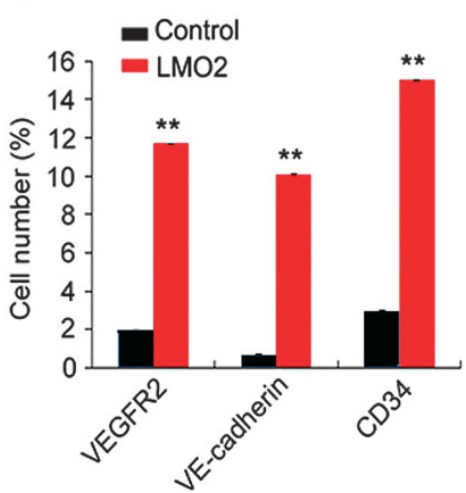

DAPI

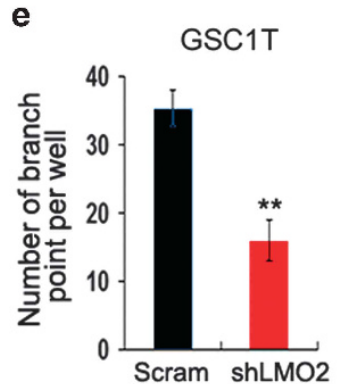

f GFP vWF DAPI

vWF

GFP
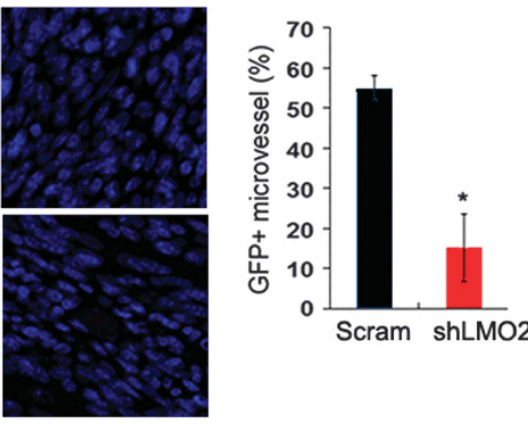

h

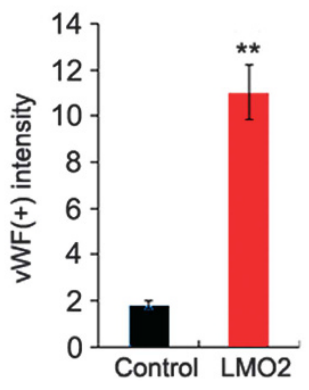

VE-cadherin promoter
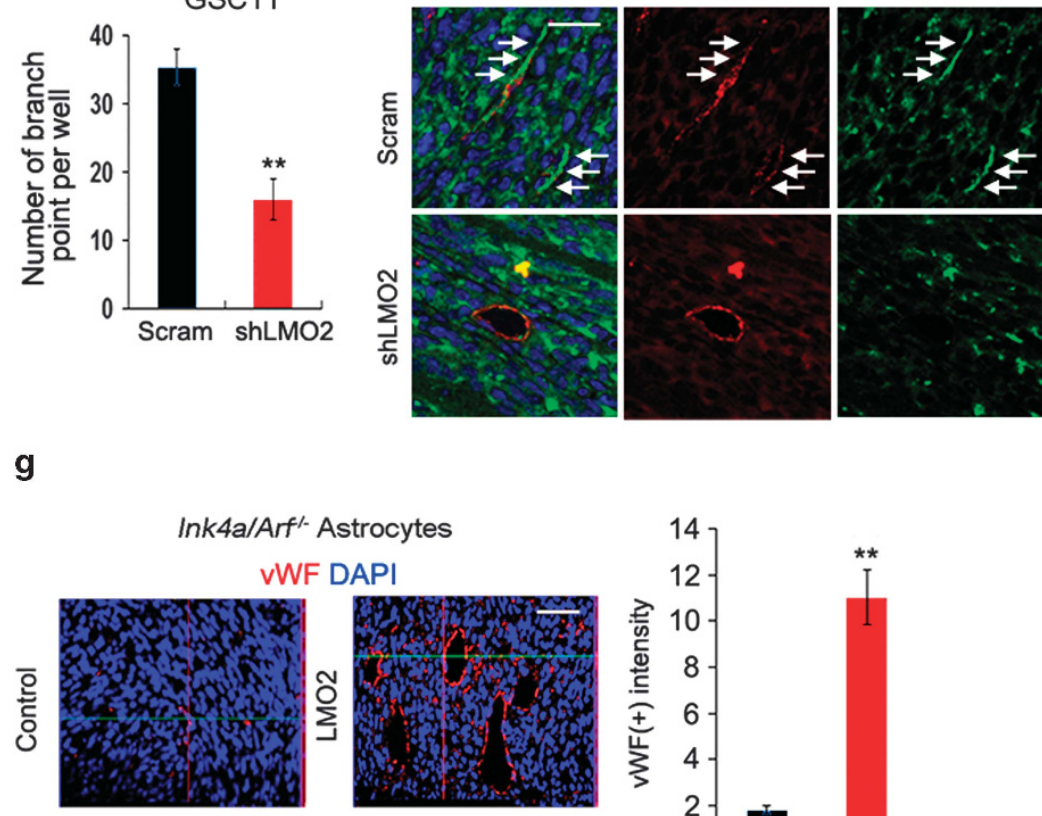

5,

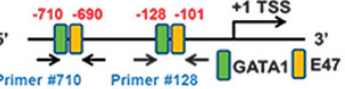

空

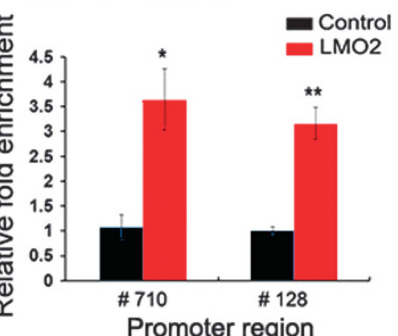

Figure 4 LMO2 modulates endothelial-like cell conversion of GSCs in vivo (a) Representative images of vWF and GFP in GFP expressing GSC-derived mouse brain tumors. DAPI for nuclear staining. Arrows indicate double-positive cells for GFP and vWF at perivascular area. Scale bar represents $10 \mu \mathrm{m}$. (b) Effects of conditioned media from controland $\mathrm{Lmo2}_{\mathrm{Ink}} \mathrm{a} \mathrm{a} / \mathrm{Arf}^{-1-}$ astrocytes on tube formation of HUVEC cells (left panel). Effect of Lmo2 overexpression on tube formation of Ink4a/Arf ${ }^{-/}$astrocytes (right panel). (c) Immunofluorescence showing VE-cadherin and VEGFR2 expressions in indicated samples. Scale bar represents $10 \mu \mathrm{m}$. (d) FACS data showing the proportions of VEcadherin(+), VEGFR2(+), and CD34(+) cells in the control- and Lmo2-Ink4a/Arf $^{--}$astrocytes grown in the proangiogenic media. (e) An in vitro tube forming ability of indicated samples. (f) GFP/vWF double-positive cells in GFP-labeled GSCs (GSC1T) brain xenografts with indicated treatments. Arrow indicates GFP/vWF double-positive cells. Graph in right panel indicates quantification. DAPI for nuclear staining. Scale bar represents $20 \mu \mathrm{m}$. (g) Representative images and quantification of the vWF(+) cells in Control- and Lmo2-Ink4a/Arf ${ }^{-1-}$ tumors. Scale bar represents $50 \mu \mathrm{m}$. (h) The occupancy of $L M O 2$ to the cognate binding elements in the VE-cadherin promoter in Ink4a/Arf ${ }^{-1-}$ astrocytes. Data are mean \pm S.D. ${ }^{*} P<0.05,{ }^{* *} P<0.01$ 


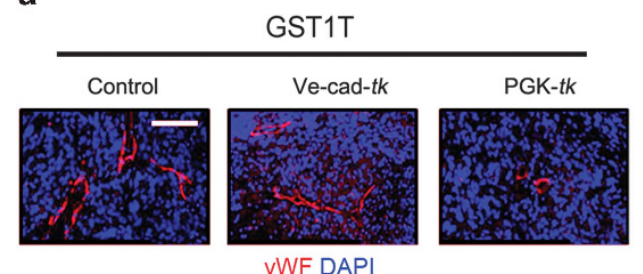

b

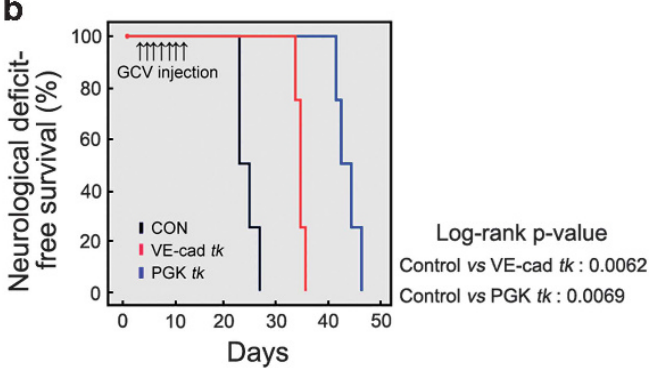

Figure 5 VE-cadherin(+) cells derived from human GSC1T give rise to endothelial-like cells and has a role in tumor growth in vivo (a) Immunofluorescence displaying vWFexpressing cells (red) in ganciclovir-treated mouse xenograft brain tumors derived from GST1T with indicated lentiviral infection. DAPI for nuclear staining. Scale bar represents $50 \mu \mathrm{m}$. (b) Kaplan-Meier curves showing the tumor-free survival of indicated tumor burden mice ( $n=4$ mice/group) after ganciclovir treatment (arrows)

IV), and clinical status of affected patients ( $<80 \%$ Karnofsky performance score; Supplementary Table 1). Collectively, LMO2 is a clinically relevant molecular target in GBM.

\section{Discussion}

The significance of this study is related to our identification of a novel function of LMO2 in GBMs and GSCs. LMO2 promotes cell proliferation and the gain of cancer stem cell phenotypes via the Jagged1-Notch signaling axis. LMO2 also facilitates endothelial-like cell conversion of GSCs by the direct transcriptional activation of VE-cadherin. Therefore, the mechanism of LMO2 action may be dependent upon spatial and cellular context. LMO2, in particular under proangiogenic conditions, resulted in endothelial cell marker expression along with subsequent endothelial-like cell conversion of GSCs. In these conditions, LMO2 might comprise a transcriptional complex with TAL-1, E47, LMO2, GATA-2, and LDB1 to regulate $V E$-cadherin in agreement with a previous study with HUVEC cells. ${ }^{18}$ In erythroid cells, LMO2 is known to bind to basic-helix-loop-helix transcription factors including TAL1/ SCL, LDB1, and GATA-1, thereby forming a large DNAbinding complex to drive the downstream target signals. ${ }^{8}$ An open question still remains as to how LMO2 coordinates to form a transcrption factor complex in order to mediate the tumorigenic activity of GSCs. Such candidates include Olig2, which is highly expressed in proneural GBM and has a critical role in maintaining stem cell phenotypes in GSCs. ${ }^{19,20}$ In support of this hypothesis, several reports have shown a striking correlation between LMO2, TAL1, and Olig2 based on gene expression analysis in human and murine T-cell leukemia. ${ }^{21-23}$ In future studies, it will be interesting to explore whether LMO2 interacts with Olig2 to regulate GSCs and brain tumorigenesis.

Presence of tumor-derived vascular components sheds light on the plasticity of cancer cells and the potential involvement of cancer stem cells in cancer-associated angiogenesis. In normal brains, Lmo2 is highly expressed in mouse vascular endothelium and is necessary for angiogenic remodeling of the existing capillary network into mature vasculature. ${ }^{17,24}$ GBMs display an enriched vasculature phenotype, which is strongly correlated with tumor aggressiveness and poor patient outcomes. Our studies presented the first evidence that LMO2 modulates endothelial-like cell conversion of GSCs by directly regulating VE-cadherin transcriptional activity. Prolonged survival of mice with the VE-cadherin promoter-driven Hsv tk suicide system further suggested the gain of the endothelial-like cell phenotype in GSCs might regulate tumor aggressiveness. Nonetheless, research on the contribution of GBM cells including GSCs to tumor angiogenesis is still evolving and many questions remain to be addressed. Recently, some studies demonstrated the evidence of transdifferentiation of some GSCs into endothelial cells, whereas others demonstrated that some GSCs are incapable of such transdifferentiation; instead they give rise to vascular stromal cells (e.g. smooth muscle cells, pericytes). ${ }^{16,25-29}$ One possible explanation for this discrepancy is that the lineage specification may be linked to the GBM/GSC subtypes. Given that neural stem cells are known to transdifferentiate into endothelial cells, ${ }^{30}$ the proneural GSC subtype may have the potential to give rise to endothelial cells rather than stromal cells. To investigate whether mesenchymal GSCs are capable of giving rise to stromal cells is also an interesting question for future studies.

In conclusion, in this study, we identified that LMO2 acts as an oncogenic transcription factor by maintaining the GSC phenotypes and inducing conversion of GSCs into endothelial-like cells. Collectively, our data provide a set of evidence suggestive of a novel signaling mechanism underlying the tumorigenic and angiogenic traits of GSCs to regulate tumor growth in vivo. Future characterization of the LMO2mediated pathways could elucidate novel molecular mechanisms of contribution of GSCs toward GBM initiation and propagation, and eventually lead to development of novel and effective targeted therapies for this devastating disease.

\section{Materials and Methods}

Ethics. Experiments using human tissue-derived materials were carried out under the approved institutional review board at Korea University, the Cleveland Clinic, and Ohio State University (OSU). All animal experimentation was performed with the approval of the OSU Animal Research Committee, following $\mathrm{NIH}$ guidelines.

Patient-derived tissue culture. GBM tissue-derived neurosphere cultures (GSC1T, GSC01, GSC13, and GSC83), GBM157, and CW3038 were established and maintained in the Kim, the Nakano, the Lathia laboratory as previously described. ${ }^{11,12}$ These GSCs were cultured in Neurobasal medium (NBE; Invitrogen, Carlsbad, CA, USA) supplemented with modified N2, B27, EGF $(20 \mathrm{ng} / \mathrm{ml}$; R\&D Systems, Minneapolis, MN, USA) and bFGF ( $20 \mathrm{ng} / \mathrm{ml}$; R\&D Systems) in suspension or in an adherent culture with laminin-coated flasks.

Cell lines and reagents. Primary HUVECs were purchased from American Type Culture Collection. Ink4a/Arf ${ }^{-/}$astrocytes were maintained in DMEM high glucose medium enriched with $10 \%$ fetal bovine serum (Hyclone, Logan, UT, USA). HUVECs and Ink4a/Arf ${ }^{-1}$ astrocytes were maintained in endothelial cell basal medium-2 with growth supplements (EGM2; Clonetics, San Diego, CA, USA). 
In vitro tube formation assay. In vitro tube formation of HUVECs, Ink4a/ $\mathrm{Arf}^{-1-}$ astrocytes, and GSCs was assessed using an in vitro angiogenesis assay kit (Chemicon, Temecula, CA, USA). Control- and Lmo2-Ink4a/Arf ${ }^{--}$astrocytes were cultured for $24 \mathrm{~h}\left(5 \times 10^{5}\right.$ cells/10-cm plate) and then used as a conditioned medium, which had been filtered through a $0.2-\mu \mathrm{m}$ filter (Sartorius Stedim Biotech, Goettingen, Germany). Primary Ink4a/Arf ${ }^{-}$astrocytes and HUVECs a

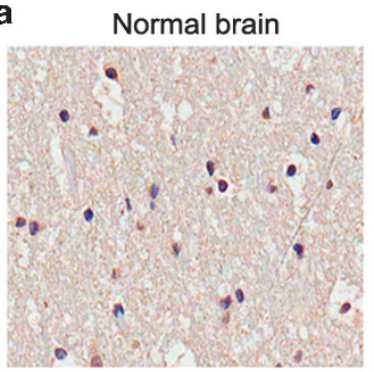

Grade II

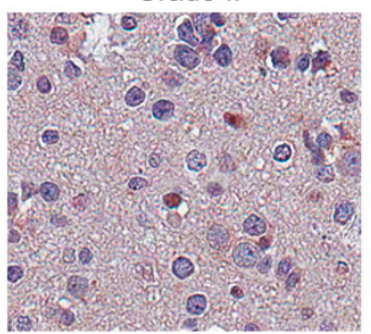

Grade III

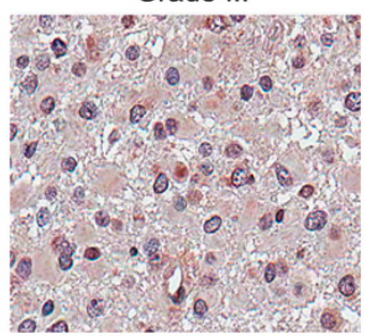

\section{Grade IV}

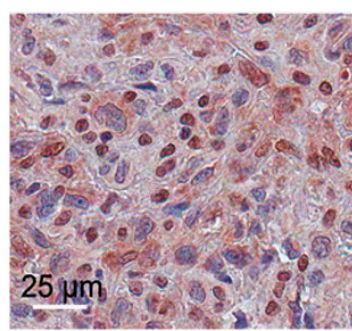

b

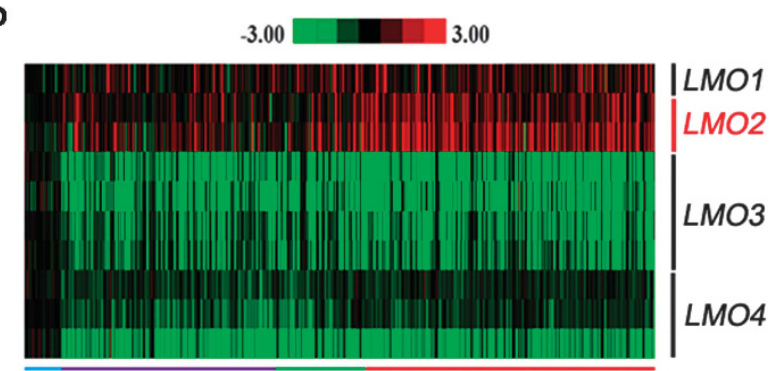<smiles>O=C=O</smiles><smiles>O=C(O)C1CCCC1=O</smiles>

c
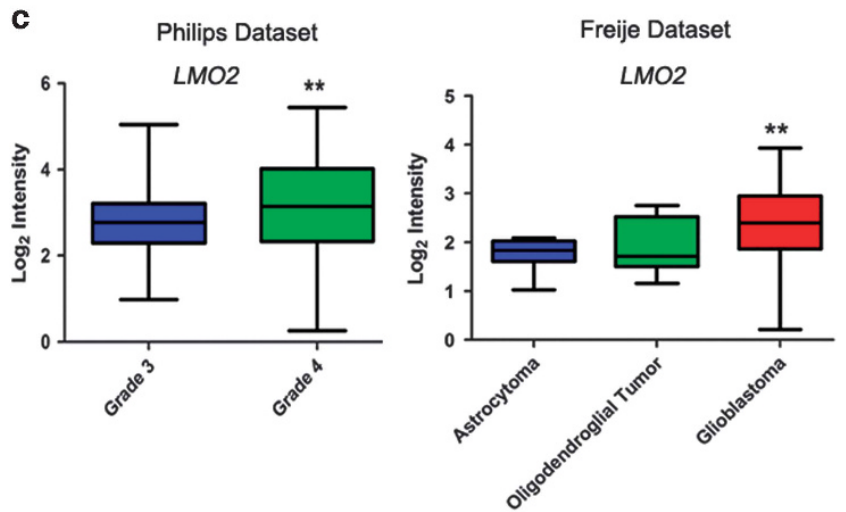

d

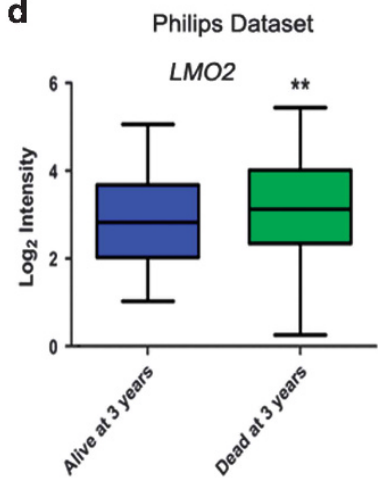

LMO2

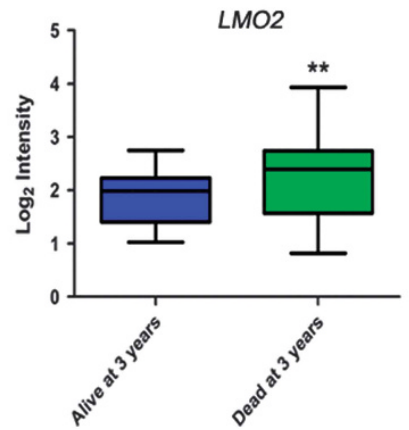

e

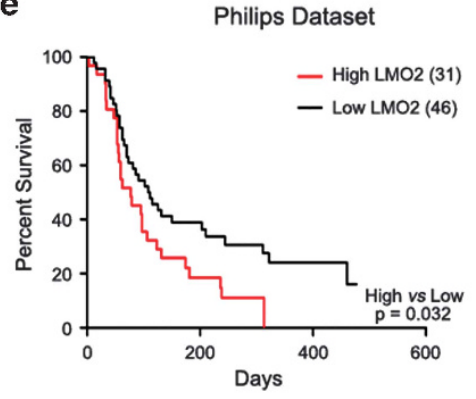

Freije Dataset
Freije Dataset

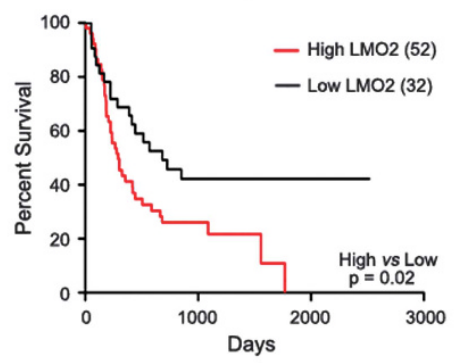

Figure 6 LMO2 is highly expressed in GBM and its expression is inversely correlated with post-surgical patient prognosis (a) Representative images of LMO2 immuohistochemistry in different WHO tumor grades of clinical glioma samples or normal brain tissues. (b) Heatmap analysis showing that elevated LMO2 mRNA levels in highgrade brain tumors. NT, nontumor; AST, astrocytoma; OLG, oligodendrocytoma; GBM, glioblastoma. (c and d) Relative expression levels of LMO2 in normal brain, astrocytoma, and glioblastoma in indicated patient groups in Phillips data set and Freije data set. (e) Kaplan-Meier survival analysis showing negative correlation of LMO2 mRNA levels with patient survival. Asterisk $\left({ }^{*}\right)$ indicates statistical significance by Student's $t$-test. ${ }^{\star *} P<0.01$ 
$\left(1 \times 10^{4}\right.$ cells/well in 96-well plate) were cultured in each type of conditioned medium at $37^{\circ} \mathrm{C}$. Six hours after incubation, the cultures were photographed $(\times 40$ magnification). Three random view-fields per well were examined, and tube numbers were counted.

Subcutaneous and orthotopic implantation assay. To establish subcutaneous xenograft models, cells $\left(2 \times 10^{6}\right)$ were subcutaneously injected into nude mice (BALB/c nu/nu mice). For the orthotopic implantation, $5 \times 10^{4}$ cells were stereotactically injected into the left striatum of nude mice (coordinates: anteriorposterior, $+2 \mathrm{~mm}$; medial-lateral, $+2 \mathrm{~mm}$; dorsal-ventral from the bregma, $-3 \mathrm{~mm}$ from the dura). For ganciclovir treatment, mice received ganciclovir at $50 \mathrm{mg} / \mathrm{kg}$ intraperitoneally for 5 days. Ganciclovir-treated mice were monitored for survival and collected samples for histology and immunofluorescence.

Statistics. Data were analyzed by two-tailed Student's $t$-test. $P$-values $<0.05$ and $<0.01$ were considered statistically significant. The correlations between gene expression levels were evaluated by Spearman's rank correlation coefficient $(\rho)$.

Other experimental materials and methods are detailed in Supplementary Materials and Methods.

Acknowledgements. We thank Drs Jeremy Rich at Cleveland Clinic, Hideyuki Saya at Keio University, Harley I. Kornblum at UCLA, and Antonio E. Chiocca at Brigham and Women's Hospital for constructive discussion and suggestions for this study. We also thank the members in the Kim and Nakano labs for constructive criticism for this paper. This work was supported in part by The American Cancer Society MRSG-08-108-01, NIH/NCI P01 CA163205, R21 CA175875, NIH/NINDS R01 NS083767, and R01 NS087913 (to IN); the National Research Foundation (NRF) of Korea grant funded by the Korean government (MEST; 2011-0017544, to HK); the Basic Science Research Program through the NRF of Korea (2011. 0024089, to SHK); and the Institute of Life Science and Natural Resources grant of Korea University (to SO).

1. Wen PY, Kesari S. Malignant gliomas in adults. N Engl J Med 2008; 359: 492-507.

2. Park DM, Rich JN. Biology of glioma cancer stem cells. Mol Cells 2009; 28: 7-12.

3. Hambardzumyan D, Squatrito M, Holland EC. Radiation resistance and stem-like cells in brain tumors. Cancer Cell 2006; 10: 454-456.

4. Rich JN. Cancer stem cells in radiation resistance. Cancer Res 2007; 67: 8980-8984.

5. Frosina G. DNA repair and resistance of gliomas to chemotherapy and radiotherapy. Mol Cancer Res 2009; 7: 989-999.

6. Schmalz PG, Shen MJ, Park JK. Treatment resistance mechanisms of malignant glioma tumor stem cells. Cancers (Basel) 2011; 3: 621-635.

7. Rabbitts TH. LMO T-cell translocation oncogenes typify genes activated by chromosomal translocations that alter transcription and developmental processes. Genes Dev 1998; 12 2651-2657.

8. Wadman IA, Osada H, Grutz GG, Agulnick AD, Westphal H, Forster A et al. The LIM-only protein Lmo2 is a bridging molecule assembling an erythroid, DNA-binding complex which includes the TAL1, E47, GATA-1 and Ldb1/NLI proteins. EMBO J 1997; 16 3145-3157.

9. Van Vlierberghe P, van Grotel M, Beverloo HB, Lee C, Helgason T, Buijs-Gladdines J et al. The cryptic chromosomal deletion del(11)(p12p13) as a new activation mechanism of LMO2 in pediatric T-cell acute lymphoblastic leukemia. Blood 2006; 108: 3520-3529.
10. McCormack MP, Young LF, Vasudevan S, de Graaf CA, Codrington R, Rabbitts TH et al. The Lmo2 oncogene initiates leukemia in mice by inducing thymocyte self-renewal. Science 2010; 327: 879-883.

11. Jin X, Kim SH, Jeon HM, Beck S, Sohn YW, Yin J et al. Interferon regulatory factor 7 regulates glioma stem cells via interleukin-6 and Notch signalling. Brain 2012; 135 : 1055-1069.

12. Mao P, Joshi K, Li J, Kim SH, Li P, Santana-Santos L et al. Mesenchymal glioma stem cells are maintained by activated glycolytic metabolism involving aldehyde dehydrogenase $1 \mathrm{~A} 3$. Proc Natl Acad Sci USA 2013: 110: 8644-8649.

13. Cancer Genome Atlas Research NetworkComprehensive genomic characterization defines human glioblastoma genes and core pathways. Nature 2008; 455: 1061-1068.

14. Singh SK, Hawkins C, Clarke ID, Squire JA, Bayani J, Hide T et al. Identification of human brain tumour initiating cells. Nature 2004; 432: 396-401.

15. Ryan DP, Duncan JL, Lee C, Kuchel PW, Matthews JM. Assembly of the oncogenic DNAbinding complex LMO2-Ldb1-TAL1-E12. Proteins 2008; 70: 1461-1474.

16. El Hallani S, Boisselier B, Peglion F, Rousseau A, Colin C, Idbaih A et al. A new alternative mechanism in glioblastoma vascularization: tubular vasculogenic mimicry. Brain 2010; 133: 973-982.

17. Gratzinger D, Zhao S, West R, Rouse RV, Vogel H, Gil EC et al. The transcription factor LMO2 is a robust marker of vascular endothelium and vascular neoplasms and selected other entities. Am J Clin Pathol 2009; 131: 264-278.

18. Deleuze V, El-Hajj R, Chalhoub E, Dohet C, Pinet V, Couttet P et al. Angiopoietin-2 is a direct transcriptional target of TAL1, LYL1 and LMO2 in endothelial cells. PLoS One 2012; 7: e40484

19. Verhaak RG, Hoadley KA, Purdom E, Wang V, Qi Y, Wilkerson MD et al. Integrated genomic analysis identifies clinically relevant subtypes of glioblastoma characterized by abnormalities in PDGFRA, IDH1, EGFR, and NF1. Cancer Cell 2010; 17: 98-110.

20. Ligon KL, Huillard E, Mehta S, Kesari S, Liu H, Alberta JA et al. Olig2-regulated lineagerestricted pathway controls replication competence in neural stem cells and malignant glioma. Neuron 2007; 53: 503-517.

21. Smith S, Tripathi R, Goodings C, Cleveland S, Mathias E, Hardaway JA et al. LIM domain only-2 (LMO2) induces T-cell leukemia by two distinct pathways. PLoS One 2014; 9: e85883.

22. Dave UP, Akagi K, Tripathi R, Cleveland SM, Thompson MA, Yi M et al. Murine leukemias with retroviral insertions at $L \mathrm{mo} 2$ are predictive of the leukemias induced in SCID-X1 patients following retroviral gene therapy. PLOS Genet 2009; 5: e1000491.

23. Ferrando AA, Neuberg DS, Staunton J, Loh ML, Huard C, Raimondi SC et al. Gene expression signatures define novel oncogenic pathways in $\mathrm{T}$ cell acute lymphoblastic leukemia. Cancer Cell 2002; 1: 75-87.

24. Yamada Y, Pannell R, Forster A, Rabbitts TH. The oncogenic LIM-only transcription factor Lmo2 regulates angiogenesis but not vasculogenesis in mice. Proc Natl Acad Sci USA 2000; 97: $320-324$.

25. Ricci-Vitiani L, Pallini R, Biffoni M, Todaro M, Invernici G, Cenci T et al. Tumour vascularization via endothelial differentiation of glioblastoma stem-like cells. Nature 2010; 468: 824-828.

26. Wang R, Chadalavada K, Wilshire J, Kowalik U, Hovinga KE, Geber A et al. Glioblastoma stem-like cells give rise to tumour endothelium. Nature 2010; 468: 829-833.

27. Ping YF, Bian XW. Consice review: contribution of cancer stem cells to neovascularization. Stem Cells 2011; 29: 888-894.

28. Soda $\mathrm{Y}$, Marumoto $\mathrm{T}$, Friedmann-Morvinski $\mathrm{D}$, Soda $\mathrm{M}$, Liu $\mathrm{F}$, Michiue $\mathrm{H}$ et al. Transdifferentiation of glioblastoma cells into vascular endothelial cells. Proc Natl Acad SCi USA 2011; 108: 4274-4280.

29. Cheng L, Huang Z, Zhou W, Wu Q, Donnola S, Liu JK et al. Glioblastoma stem cells generate vascular pericytes to support vessel function and tumor growth. Cell 2013; 153: 139-152.

30. Wurmser AE, Nakashima K, Summers RG, Toni N, D'Amour KA, Lie DC et al. Cell fusionindependent differentiation of neural stem cells to the endothelial lineage. Nature 2004; 430 : 350-356.

Supplementary Information accompanies this paper on Cell Death and Differentiation website (http://www.nature.com/cdd) 